\title{
BMJ Open Australian pharmacists' perspectives on physician-assisted suicide (PAS): thematic analysis of semistructured interviews
}

\author{
Sami Isaac (D) , ${ }^{1}$ Andrew McLachlan, ${ }^{1,2}$ Betty Chaar (D) ${ }^{1}$
}

To cite: Isaac S, McLachlan A, Chaar B. Australian pharmacists' perspectives on physician-assisted suicide (PAS): thematic analysis of semistructured interviews. BMJ Open 2019;9:e028868. doi:10.1136/ bmjopen-2018-028868

- Prepublication history and additional material for this paper are available online. To view these files, please visit the journal online (http://dx.doi. org/10.1136/bmjopen-2018028868).

Received 05 January 2019 Revised 09 September 2019 Accepted 11 October 2019
Check for updates

(C) Author(s) (or their employer(s)) 2019. Re-use permitted under CC BY-NC. No commercial re-use. See rights and permissions. Published by BMJ.

${ }^{1}$ School of Pharmacy, Faculty of Medicine and Health, The University of Sydney, Sydney, New South Wales, Australia ${ }^{2}$ Centre for Education and Research on Aging, Concord Repatriation General Hospital, Sydney, New South Wales, Australia

Correspondence to

Sami Isaac;

sami.isaac@sydney.edu.au

\section{ABSTRACT}

Objectives This study aimed to investigate Australian pharmacists' views about their role in physician-assisted suicide (PAS), their ethical and legal concerns and overall thoughts about PAS in pharmacy.

Design Semistructured interviews of pharmacists incorporating a previously validated vignette and thematic analysis.

Setting Australia (face to face or phone call).

Participants 40 Australian Health Practitioner Regulation Agency registered pharmacists, majority women (65\%) with varied experiences in community, hospital, industry, academia, government and other fields.

Results Emergent themes from the interviews were: legal and logistical framework, ethical framework, training and guidance and healthcare budget. More than half the participants supported the role of pharmacists in the supply of medicines for PAS, while less than half were either against or unsure of the legislation of PAS in Australia. Shared concerns included transparency of prescribing practices and identification of authorised physicians involved in PAS, which were consistent with existing literature. Religious faith, emotion and professional autonomy were key indicators for the implementation of conscientious objection to the supply of medicines in PAS. Re-evaluation of current guidelines, pharmacist training and government reimbursement was also of significance from participants' perspectives.

Conclusion This study revealed current concerns of practising pharmacists in Australia, including previously undocumented perspectives on the pharmacoeconomic impact of and barriers relating to PAS. The need for training of all healthcare professionals involved, the provision of clear guidelines, including regulation around storage, administration and disposal of medicines dispensed for PAS and the updating of current therapeutic guidelines around end-of-life care were all issues delineated by this study. These findings highlighted the need for current and future policies to account for all stakeholders involved in PAS, not solely prescribers.

\section{INTRODUCTION}

The right to 'die with dignity' has been legalised across select countries under differing conditions. ${ }^{1}$ Belgium, Netherlands and Luxembourg have legalised both euthanasia and

\section{Strengths and limitations of this study}

- This study undertook an integrated qualitative approach with semistructured interview and thematic analysis of data to capture in-depth detail of the pharmacist's views on physician-assisted suicide (PAS) - something that may not be achieved with conventional quantitative methods.

- This is the first study to provide insight into the perspectives of Australian pharmacists on PAS, which could be used to further future studies and help shape policies and legislative frameworks.

- Despite interviewing participants from various locations across Australia and reaching saturation, a broader diversity of participants could have provided more comprehensive data of the entire international pharmacy profession.

- Video recording of the interviews may have also strengthened the study through paralinguistic analysis of captured non-verbal cues.

physician-assisted suicide (PAS). Columbia, Canada, Switzerland (under specific laws) and the US states of Oregon, Washington, Vermont, Montana, Hawaii and California $^{23}$ and the Australian state of Victoria (as of 2019) have legalised PAS.

For the rest of the world, it remains illegal. However, the debate around this topic is becoming more challenging as populations age, and there is a growing public interest in PAS. Healthcare providers-including pharmacists-need to be actively involved in this development, and be prepared to use international experiences and precedence to help establish boundaries and guidelines that protect all those involved in PAS.

The definitions around end-of-life vary across literature and different countries. They are primarily based on terminology used in legislation drafted in a particular country. However, the Oxford dictionary defines euthanasia as 'the painless termination of a life-by some medical means ${ }^{4 \text {, with }}$ 
the subdivisions, voluntary and involuntary euthanasia. As per international laws, 'euthanasia' is limited to voluntary cases, where the individual is mentally competent and explicitly requests euthanasia. Involuntary or nonvoluntary euthanasia is when euthanasia is undertaken without explicit patient request or consent. ${ }^{5}$

In PAS, a physician provides access or means by which a competent person can choose to self-administer a lethal dose of medication (typically barbiturates) with the intention of ending their life. PAS achieves the same outcome as euthanasia with a clear distinction in the method by which that outcome is achieved. In PAS, the person selfadministers the fatal dose, while in euthanasia another individual (usually a physician) administers the dose. ${ }^{6}$ Other terms used in end-of-life/palliative care include the absence, withdrawal or refusal of life-saving treatment. In many countries, including the USA and Australia, it is deemed legal and ethical when such decisions are made with the patient's or proxy's agreement. Such practicesreferred to as 'advance directives ${ }^{7}$ ' - are not characterised as euthanasia per se, but rather form part of common law, 'governing the doctor-patient relationship and the provision of medical treatment more generally'. ${ }^{28}$

Two studies, conducted in Australia, ${ }^{9}{ }^{10}$ found that only 45 per cent of sampled physicians supported the legalisation of euthanasia, and only under certain circumstances. Another study in 2007 reviewing physicians' opinions concluded that views across the profession 'are sharply polarised and need further debate. ${ }^{11}$

Nurses' views have also been investigated, with a 2018 study finding most nurses perceived PAS as an extension of their professional practice, while a small number expressed moral distress regarding assisting death. ${ }^{12}$ Another Finish study reported that 75 per cent of nurses were willing to participate in PAS if it were legal. ${ }^{13}$

Despite literature exploring physician and nurse perspectives, very little research has been conducted exploring pharmacists' roles and opinions on this issue. In Organisation for Economic Cooperation and Development countries, ethical principles in medicine mandate a degree of separation between prescribing a drug and its supply, thus necessitating the need for independent channels of distribution (ie, pharmacists). Thus, pharmacists have the responsibility of stocking, storing, handling and overseeing the ethical supply of medicines, including those prescribed for PAS.

Like other clinicians, pharmacists are therefore morally implicated and have a duty of care to be involved and aware of their patient's health outcomes. Additionally, pharmacists are regarded as 'experts in medicines' and an independent checkpoint in the provision of medications; with adequate qualifications and expertise, their role expands to one which serves the community in providing healthcare advice as part of the primary healthcare team. The inclusion of pharmacists' views enhances the development of thorough and robust health policies that ensure safe and appropriate use of medicines, including those used in PAS.
This study aimed to investigate Australian pharmacists' views on PAS, its legislation and their role in the supply of medicines to people seeking PAS.

\section{METHODS}

\section{Recruitment}

All participants provided written informed consent prior to participation. This study incorporated a snowball sampling strategy, with the circulation of invitations via word of mouth and advertisement in professional society's newsletters and on social media platforms (Facebook and Twitter). The only inclusion criteria were that interviewees were currently registered with the Australian Health Practitioner Regulation Agency as pharmacists, and willing to express their views on the legalisation of PAS. Recruitment was initiated via email and interviews conducted via phone or face to face. Participants had no prior knowledge of the interviewing researcher other than what was provided (electronically or hardcopy) on the invitation, consent and participant information forms (online supplementary material). A broad range of locations around Australia were targeted to capture a variety of perspectives based on location, practice environment and experience in pharmacy including but not limited to community, hospital and industry. Professional organisation representatives were classified as such based on their credentials, role in the Australian pharmacy profession and their ability to influence the industry as professional leaders and representatives.

\section{Researchers' characteristics and reflexivity}

All researchers (SI, AM and BC) are registered pharmacists and academic members of The University of Sydney, School of Pharmacy, Faculty of Medicine and Health. SI is a $\mathrm{PhD}$ candidate and MD student. $\mathrm{AM}$ is a Professor, Head of School and Dean of Pharmacy. BC is an Associate Professor at the School of Pharmacy.

\section{Interview design}

An interview protocol (online appendix table 1) was developed based on research literature on PAS $^{14}$ and the study objectives. The semistructured interviews incorporated a previously validated vignette ${ }^{14}$ used as a tool to initiate the interview conversation, followed by openended questions to enable the exploration of new ideas, with prompts to allow deeper probing and expansion of critical issues relating to PAS.

\section{Thematic analysis}

For uniformity, the interviews were conducted by one interviewer (SI) from September to November 2017 and were on average at least $30 \mathrm{~min}$ in duration. Interviews were audio recorded, deidentified and transcribed ad verbatim. Data were then uploaded into NVivo (QSR V.11.0.0 Mac) software for thematic coding by SI. For quality control, the research team (SI, BC and AM) independently read and coded transcripts into themes. These themes were 


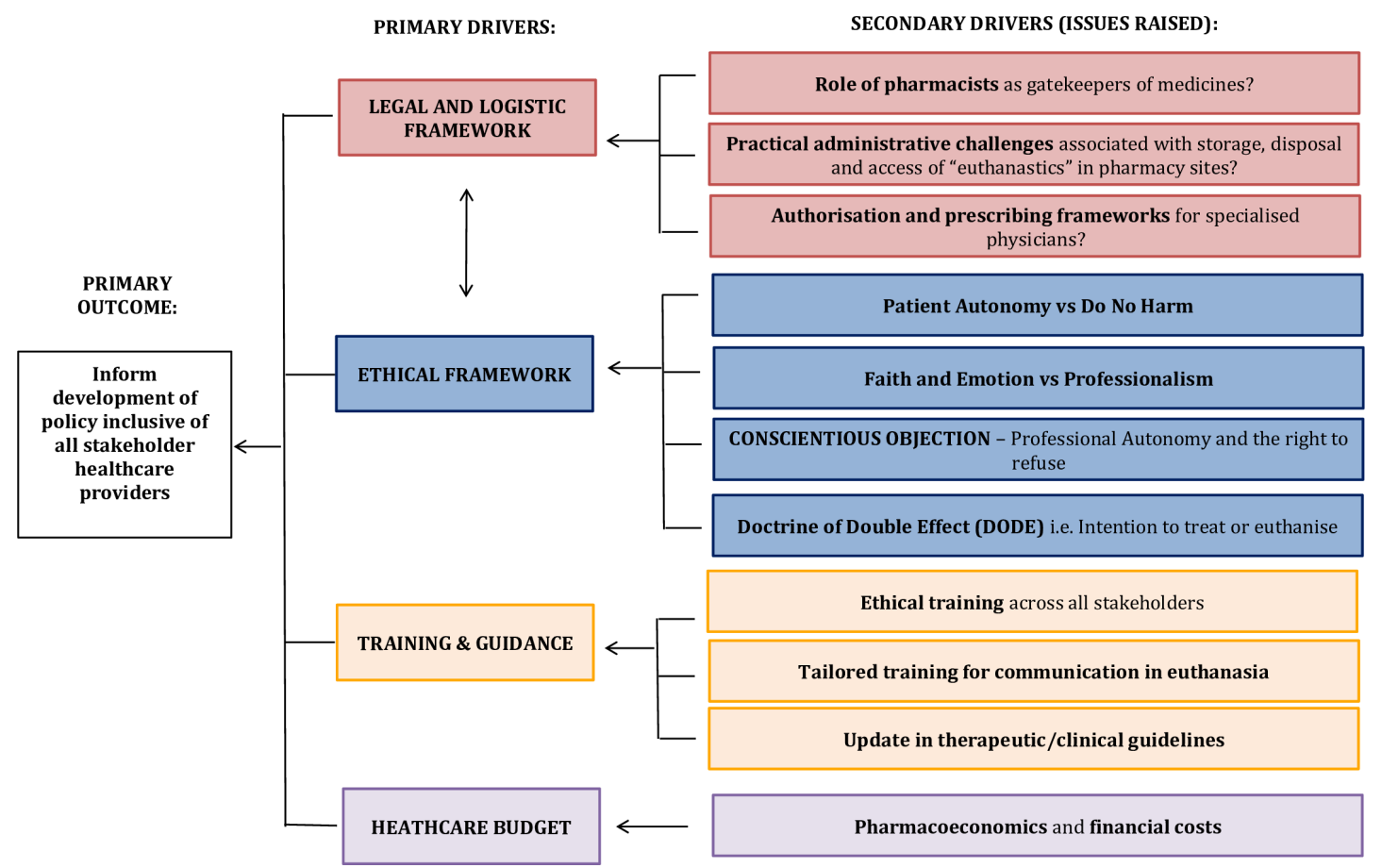

Figure 1 Driver diagram of emergent themes from interviews $(n=40)$.

discussed and reviewed until all authors agreed on the coding scheme from the thematic analysis..$^{15}$ Data saturation was reached after the analysis of 35 interviews; however, additional interviews were analysed to assure that there was no new information before concluding the data were saturated and terminating data collection after 40 interviews. Saturation was determined as the point in which no new thematic codes became emergent during analysis. The analytical technique of constant comparison (a component of grounded theory) ${ }^{16}$ was used to help extract key perspectives and ensure a level of consistency and reliability of analysis. Data analysis was conducted following the COnsolidated criteria for REporting Qualitative research (COREQ) checklist and these key themes were then used to generate a driver diagram (figure 1).

\section{Patient and public involvement}

Other than being recruited and interviewed (as indicated above), the participants of this study were not involved in the design or development of the study.

\section{RESULTS}

\section{Participant demographics}

A total of 40 respondents, from across seven states or territories of Australia, who met inclusion criteria, participated in the study. The majority were women, with varied levels of practice experience (online appendix table 2) and primary roles such as: community, hospital, industry, government, academia and pharmacists from professional organisations.

Pharmacists who voluntarily indicated religious influences on their decision-making expressed concerns and reservations about pharmacists' role in PAS. Others, mainly those who had past personal experiences of terminal illness with family members, expressed being comfortable with the notion of PAS. The majority of pharmacists who participated in this study reported they were in favour of PAS if supported with a clear legislative framework and clinical evidence of efficacy.

One pharmacist encapsulated the views identified across this study by stating:

Pharmacists are a very diverse group with varied views when it comes to political voting, whether it's left or right. But when scientific evidence comes into things, that's where we rally around objective truths or as close to objective truths as we can get... I think that the pharmacy perspective and the pharmacy industry as a whole, will be more or less supportive of this.

(Pharmacist 16)

The results of the thematic analysis of the interviews were presented under the following four emergent themes:

1. Legal and logistic framework.

2. Ethical framework.

3. Professional training, and guidance.

4. Healthcare budget.

These themes were triangulated to generate a driver diagram (figure 1) which captured findings to showcase sociopolitical drivers around PAS from pharmacists' perspective. It is a conceptual map of themes emerging from ideas expressed by participants in this study and helps translate the primary goal of informing future studies and the development of policy to include all stakeholders' perspectives, particularly pharmacists', into a logical set of underpinning themes or drivers. 


\section{Driver 1: legal and logistic framework}

Most participants agreed that pharmacists play an essential role in the supply of medicines involved in PAS. Known as the 'gatekeepers of medicines', pharmacists perceived their role as a central checkpoint necessary to ensure appropriate prescribing in addition to communicating with patients and carers as part of an interdisciplinary healthcare team.

I'm not comfortable with the idea of the doctor giving out the drugs, as well as prescribing them. It is a real possibility they could get use to prescribing PAS as an easy way to kind of help the elderly and not look at other options. I guess that's why the gatekeeper role of the pharmacist would be useful to help minimise that risk (Pharmacist 37)

It should be a multidisciplinary team. It shouldn't really be based on one doctor's opinion. It should be the oncologist, doctor, psychiatrist, pharmacist etc. It should be a team of palliative care staff who make the decision. (Pharmacist 19)

Some participants identified practical concerns associated with supplying medicines for PAS to a patient to administer at home. This included appropriate storage, access by other people and appropriate disposal of medications used in PAS.

I would prefer it to happen in a seamless process at that time. I'd be worried that the medication will just sit in the cupboard and a partner or child accidentally takes that medicine... As a pharmacist, we see the risks of polypharmacy and stocking of medicines. I'm just concerned about that. (Pharmacist 2)

Also highlighted was the fact that pentobarbital (Nembutal), a drug that has international precedence for PAS, is currently illegal for human use in Australia.

I think it is reproachable that we cannot follow global precedence to follow what has been used, for no reason other than stubbornness from the powers that be. I don't see why they are not legalising it... it seems that animals have more rights than humans in this country. (Pharmacist 7)

This statement illustrates the variation in legislation and drug selection, and the need for closer evaluation of the drug/s of choice and their effects versus risks profile. In Australia as it stands, a compounded oral preparation combining various legal medications is the only available method for PAS. This in itself opens an ethical mind field in regards to identifying readily used prescription medicines for PAS.

Participants expressed the need for strict protocols, clearly explaining the requirements around drug storage, administration and disposal, to be transparent and enforceable. Also, the clarification of liability once the medication is in possession of the person or their carer would need to be made.
The need for stricter drug classification protocols and standardisation of medication used in PAS, as well as clear identification of a prescriber's authority and intentions, were legal requirements also considered of high priority for the majority of pharmacists in this study.

The prescription would need to be endorsed with 'PAS' for instance... a register of medical physicians authorised to implement PAS would also be needed. It should be electronic and enable pharmacists to verify and validate that they are physicians who have been trained and allowed to provide such a treatment option to terminal patients. I feel the profession would need to safeguard this process if it were to be legalised. (Pharmacist 24)

Having healthcare professionals listed on a national database was suggested as a possible solution to identify prescribers' expertise and intentions for PAS. Some respondents suggested a restriction to access to medicines used in PAS as a safeguard to mitigate concerns for storage, prescriber identification and misuse.

I think patients should be under medical supervision during the process of physician-assisted-suicide. It should be some kind of clinic where they can be registered to die in peace, not just ad hoc at home in the kitchen or something. (Pharmacist 7)

These concerns, expressed by many participants repeatedly, reflected a shared fear of legal loopholes, legislative uncertainty and desire for a strict, conservative approach to PAS implementation, one which can ensure safety, reduce liability and avoid errors.

\section{Driver 2: ethical framework}

\section{Conscientious objection}

At the core of the ethical framework theme is the notion of conscientious objection, defined as a pharmacist's right to decline to supply a medicine or service, based on personal beliefs, faith, emotion or the need for professional autonomy. An ethical dilemma expressed by several participants was about healthcare professionals' right to refuse supply of medicines for use in PAS (conscientious objection) in balance with respect for patient autonomy.

I don't believe that it's our place to decide when we die; God decides that. (Pharmacist 14)

If PAS is enacted, there won't be a life to try and improve the quality of, because they would be dead. (Pharmacist 33)

Some participants felt strongly against the notion of faith-based conscientious objection. They viewed it as an unnecessary obstacle and restriction to medical advancement, some more emotionally charged than others.

It's always the religious zealots who think PAS is against the Bible. I think that's the biggest challenge. (Pharmacist 35) 
For a few pharmacists, the emotional burden was in itself identified as a key marker for conscientious objection. The sheer magnitude of the notion of PAS and ending another human's life-irrespective of it being autonomous or not-was for some, too emotionally burdening.

Even though I support PAS, I can tell you if I was sitting at home right now knowing the drugs I supplied will be used to kill somebody, I would be an emotional wreck. (Pharmacist 2)

Most pharmacists proposed an 'opt-in' policy for pharmacists to allow for professional autonomy yet ensure adequate access to those who seek PAS.

I think it's important it's "opt-in" ... there is always going to be conscious objectors, and they are absolutely entitled to that. That's why I think an opt-in process is probably the safest, most conservative way to do this. It just makes sense. (Pharmacist 37)

\section{Doctrine of double effects}

The complex medical principle known as the doctrine of double effect (DODE) was mentioned on multiple occasions by pharmacists who felt PAS already takes place in palliative care to a certain extent. DODE is a philosophical principle that has been embedded in medical law ${ }^{17}$ where a doctor who acts intending to achieve a primary effect which is good, does not intend and is not culpable of a bad secondary effect. This could apply to the case of palliative care practices where the provision of pain relief does not make the physician culpable for hastening the patient's death through respiratory depression. ${ }^{18}$

People are already dying through dehydration and starvation because they are classified as 'Nil by Mouth'. They literally just fade away in the bed over a week. That's not even dignified in any way. It's just mean...it's cruelty! Because really you could put a drip for fluids in IV (sic), but then they could last for 3 months, unable to rehab because they are too old. But who makes that decision? And opioid doses are so high in these patients that there is just no way they do not get respiratory depression. (Pharmacist 9)

Participants also identified limitations to palliative care services, and the perceived ability for PAS to complement palliative care processes. Participants suggested that palliative care and PAS could be implemented side by side as a holistic and autonomous approach to end-of-life care.

Palliative care isn't like you sit in God's waiting room, and everything is all nice and great. It's an unpleasant process and the reason why we still need PAS (Pharmacist 3)

Many participants felt that a profound ethical dilemma arises from introducing such a challenging medical procedure as PAS, primarily due to the need to stop suffering and the innate human fear of death.

.... there are a lot of very polarised views of people who really believe in their side, whether for or against. It's okay to hold those views, but if we look at it from a health-professional point of view and a patientcentred practice, then to give them this option, just because we have legislation, doesn't mean everyone is going to take this option, right? (Pharmacist 8)

The above quote is one which identifies the complexity around ethics, in particular bioethics of end-of-life care. The uncertainty around the implementation of PAS is carried through by how the pharmacist posed their statement as a question, highlighting the inherent hesitation around the implementation of such policy, despite having international precedence.

\section{Driver 3: professional training and guidance}

Some pharmacists acknowledged a lack of extensive understanding about PAS, primarily due to its legal status in Australia.

I haven't heard it being brought up in any topic and among not just pharmacists, but doctors and nurses and other health professionals. So maybe it is a topic that is not spoken about often (Pharmacist 33)

Drugs used for PAS were not differentiated from other drugs on the market. Training would need to be centred on the optimal dose of the medicine and its effects, and on person-centred information around counselling, emotional strategies and legislative requirements.

Look I don't think it will be rocket science... other than dealing with the sensitivities of the communication... But pharmacists have adapted to a whole load of ethical issues or changes over the years (Pharmacist 9)

The majority of participants suggested the need for the development of new training courses and learning opportunities to ensure a greater understanding of PAS. They also reiterated a shared concern for ensuring that the medications successfully achieve the desired outcome after the first administration.

Will there need to be a new set of guidelines for doses? What is going to adequately kill you vs me is different. It would depend on weight, metabolism, tolerance all those types of things. And who is going to be monitoring and recommending all these values and doses? It is going to be extremely intricate and the key would be to get it right the first time if they are going to implement it. (Pharmacist 26)

\section{Driver 4: healthcare budget}

Several participants indicated concerns about drug cost/s and whether the government would subsidise the process, 
and if so, to what extent-as this would impact on medicine accessibility and supply protocols.

What about the cost of the medication being used. Will it be on the PBS (The Pharmaceutical Benefits Schedule (PBS) provides Australians with subsidised access to a selected list of medicines) or private? Would it be really expensive? Can the hospitals or the government cover the cost? (Pharmacist 27)

Other concerns were raised regarding the distribution of financial support across other end-of-life care fields, such as palliation and age care services. ${ }^{19}$

As a society, we have a responsibility to potentially look at what (sic.) other ways we could avoid these situations occurring. Another aspect that I see is aged care in the home, and a lot more resources should be placed on aged care in the home (Pharmacist 24)

Interestingly, in addition to costs of medicines, pragmatic aspects to end-of-life care were also raised about the healthcare budget and pharmacoeconomics surrounding the prolongation of life in the presence of the notion of 'futility'.

The way the healthcare budget is as it is, we need to look at things pragmatically. And realistically if you can determine that somebody is coming towards the end of their life I don't think you should prolong it unnecessarily. (Pharmacist 24)

This issue was raised by many pharmacists and highlighted an important aspect about the concept of medical futility and the cost related to extending life when the endpoint was determined to be futile. The honest yet difficult opinion on the broader picture of this topic is a relatively new concept that has not been presented by pharmacists before in the literature. Pharmacist 24's views highlighted the growing economic dilemma related to a globally ageing population, and the problems associated with prolonged healthcare costs alongside steady decline or complete lack of quality of life.

These differing perspectives identified the new emergent and relevant theme of cost and healthcare budget, which is fundamentally at the core of many developed nations health initiatives.

\section{DISCUSSION}

\section{Statement of principal findings and comparison to existing literature}

This study found that the majority of pharmacists interviewed supported their role in the supply of medicines for PAS. Some respondents expressed concerns about the uncertainty of the legal aspects of PAS in Australia, including concerns about access to pentobarbital (Nembutal) already used in other countries around the world. Respondents agreed that there needed to be transparency in prescribing practices and clearer identification of authorised physicians involved in PAS. Conscientious objection to PAS was considered a professional right by most pharmacists and this was related to religious faith, emotion and professional autonomy. Participants called for clear guidelines to inform implementation of PAS and training to enable pharmacists to take their role in provision of this service. Economic and pragmatic concerns were also raised about costs of prolonging life in futility when no quality of life was expected.

\section{Driver diagram-framework guidelines}

In order to provide a framework for clear guidelines, we have amalgamated the four emergent themes derived from the interviews into a driver diagram (figure 1).

The four emergent themes derived from the interviews highlighted the need for a more explicit legislative and ethical framework, supported by quality training in both clinical and behavioural aspects of delivering PAS. To inform the development of guidelines, the driver diagram was developed to help translate a high-level improvement goal into a logical set of underpinning goals and projects. It captures an entire change programme in a single diagram and provides a measurement framework for monitoring progress. The following were the drivers for the framework devised.

\section{Driver 2: ethical framework}

The key driving theme was the need for creating a robust foundational ethical framework and training guide for the safe implementation of PAS. For many, an ethical framework that emphasised respect for both patient autonomy and their right for choice in life and death was counterbalanced by requests to preserve pharmacists' professional right to conscientious objection. Conscientious objection has always been a point of contention and debate for many healthcare professionals. By definition, it is a professional's 'right to refuse to engage or provide a service primarily because the action would violate their deeply held moral or ethical values about what is right and wrong. ${ }^{20}{ }^{21}$ Similar to the ethical dilemmas associated with the provision of the emergency contraceptive pill or the abortifacient combination medication RU486 (M2-Step), ${ }^{22}$ the provision of PAS involves pharmacists and warrants that their views be considered. Most participants reiterated this concern and responded per their code of ethics. ${ }^{23}$ Irrespective of any refusal to participate in PAS, or provision of the emergency contraceptive pill or any other ethically challenging procedure, there will always be the need for continuity of care. When invoking one's right to conscientious objection, it goes without saying that ensuring basic professional standards are preserved is paramount, and this by no means warrants an attempt to influence the patient's views. It is the enactment of mutual respect for different perspectives, which can be resolved professionally through referral to another healthcare professional.

The influence of religion and faith were prominent among many, whose perspectives were clearly influenced by their religiosity. This is in line with literature, ${ }^{24}$ which highlights that a multitude of factors, including faith and 
ethical predispositions, can affect an individual's judgement, even as a healthcare professional. Verweel et $a l \mathrm{~s}^{25}$ analysis of Canadian guidelines identified the notion of conscientious objection in some, but not all of the guidelines, and no mention of the concept in Canadian federal legislation. This absence of an ethical framework in legislation was identified by our participants as a key issue, with a request that legislation stipulates pharmacist's options (Pharmacist 37, p11), rights and responsibilities. This is echoed by Hanlon $e t$ al and Berard and Walker who identified that pharmacists believed that any federal legislation that protects physicians' freedom of conscience should apply equally to pharmacists. ${ }^{1426}$

\section{Driver 1: legal and logistic framework}

Others were concerned about pharmacists' liability and the lack of involvement as seen in international precedence. The US state of Oregon's Death with Dignity Act neglected the role of pharmacists in the original statute (1994) stating that pharmacy practice includes 'participation in drug selection and drug utilisation reviews' and 'responsibility for advising of therapeutic values, content, hazards and use of drugs and devices'. ${ }^{27}$ But in 1997, Oregon amended its statute to require physicians to inform pharmacists of the purpose of a lethal medication dose in advance. ${ }^{28}$

In the Netherlands, despite being one of the first countries to adopt euthanasia, the Dutch Termination of Life on Request and Assisted Suicide Act, ${ }^{29}{ }^{30}$ to this day, fails to refer to the role of pharmacists explicitly. This lack of pharmacists' role in legislation is also apparent in Belgium, which shares Dutch laws. ${ }^{31}$ Switzerland also fails to recognise pharmacist involvement and in fact has minimal physician supervision, instead relying solely on PAS organisations such as EXIT or Dignitas to provide the service. ${ }^{29}$ Therefore, concerns about the uncertainty behind legal and logistical frameworks were a primary driver. There were repeated requests for clarity in acknowledging and defining the role of pharmacists in PAS, as well as guidance in regards to medication storage, disposal and communication strategies. This was in keeping with literature ${ }^{25}$ which has identified recommendations of guidelines to 'pharmacist's role in quality assurance' including preparation, storage, destruction and documentation of the PAS process.

\section{Driver 3: professional training and guidance}

To prevent uncertainties, the need for greater awareness and training emerged as an additional primary driver. Majority of participants described a need for professional training and establishing new guidelines (both therapeutic and practice based) to allow a seamless and robust implementation process of PAS. Many proposed that the existing representative professional bodies be involved in ensuring that these protocols protect pharmacists legally and professionally and are comprehensively reviewed and updated regularly.
Driver 4: healthcare budget

Finally, concerns about financial issues and costs were unique to the findings of this current study. Questions were posed about the pharmacoeconomics associated with implementing PAS. The concept of futility was also introduced within this context, with participants raising doubts about the draining costs of prolonging life pointlessly.

Over two decades ago, this issue was raised in the literature by Emanuel and Batton ${ }^{32}$ highlighting two opposing perspectives on this conundrum. On one hand, there are recognisable cost savings to be had with PAS, while on the other hand financial incentive can cause ethical concerns associated with family members resorting to PAS to spare their families the substantial economic burden of endof-life healthcare costs. Some of the amicus curiae brief (expert documents) submitted to the Supreme Court speculated that cost savings made by implementing PAS are 'as undeniable as gravity... the earlier a patient dies, the less costly his or her care is ${ }^{33}$ In our study, we found that several participants highlighted that due to the ongoing burden of cost on our healthcare system, PAS needs to be considered as a viable option for the terminally ill. Despite being morally controversial, understanding the economic impact of PAS is important in determining the financial implications of the practice and whether or not it will be supported by government or private funding.

\section{Strengths and weaknesses of the study}

Given the global discussion about PAS as a contemporary bioethical debate, this study fills a gap in the literature when it comes to investigating the Australian pharmacy profession's detailed perspectives and concerns regarding PAS. A strength of this research is its qualitative nature, which allows pharmacists' personal and professional concerns to be probed and explored in-depth.

Some limitations of this study included the diversity of participants interviewed, which may not be generalisable to the entire international pharmacy profession. However, data saturation was ensured with the analysis of an extra five interviews beyond thematic saturation, and this was to ensure no new information was identified before concluding the data to be saturated and terminated at 40 interviews. An even broader sample size would have been ideal; however, as indicated in our methods, we did not use a purposive sampling strategy due to the limitations associated with that sampling style. Instead, we used a snowball sampling strategy which was the most approached given the context of interview working pharmacists and yielded the sample size and demographics (online appendix table 2). Video recording of the interviews may have also strengthened the study through paralinguistic analysis of captured non-verbal cues.

\section{Strengths and weaknesses in relation to other reviews}

In the year 2000, a Dutch study showed that most pharmacists have in some way been directly affected by PAS during their careers. ${ }^{34}$ In this study, 78 per cent of community 
pharmacies had received at least one request to dispense drugs for PAS, and 11 per cent received between 6 and 10 requests. ${ }^{34}$ The same research also found that 88 per cent of hospital pharmacies had received at least one request to dispense these medicines. ${ }^{34}$

Despite pharmacists' active involvement in the legal supply of drugs for PAS, little research and legislative policies are detailing the role of pharmacists. In the Netherlands, there have been some commentary articles recently published in the Lancet highlighting the moment Australia's state of Victoria passed the bill to legalise PAS $^{35}$ and another reviewing the trends in Netherlands and Belgium. ${ }^{31}$ In the Netherlands, it is essential to identify the time disparity between the practice of euthanasia (as is most commonly practised) and its official legislation, which was passed 2 years after the practice started.

Other international studies ${ }^{14213436-40}$ of a similar sample collected data using a quantitative approach (questionnaire), which in most cases did not capture the rich data attained in our study through semistructured interviews. Their findings focused on the percentages of pharmacists who were for or against PAS, with the additional question of whether or not an individual has the right to choose their own death. These questions were commonly asked in a Likert scale format which provided quantitative yet dichotomous insight into pharmacists' views on conscientious objections and frequency of exposure to PAS prescriptions. The exception was that of Hanlon et $a l^{14}$ which implemented a mixed-method approach developed from a series of qualitative interviews and guided by vignettes (employed in this study). The results of this study showed similarities to the UK paper, particularly around pharmacist's views on conscientious objection, which is grounded in the need for legislative protection of conscience in line with that provided to physicians. The dilemma of bioethical pillars and legislative concerns were also shared findings, highlighting similarities in pharmacists' views from the UK and Australia. Contrastingly, the UK study ${ }^{14}$ did not identify the issues of healthcare pharmacoeconomics, logistical need for tailored training and update in therapeutic and clinical guidelines as emergent themes. This relates to the importance of cost to the patient, pharmacy and healthcare system as influential factors that have a bearing on healthcare changes, including PAS. Some articles did draw associations between demographics (such as age and sex) and responses, but almost all failed to identify why their participants answered the way they did, and the rationale and understanding of the question posed to them. The policy implications of most of the articles' findings were on pharmacists' moral and legal implications in PAS and the level of pharmacist involvement. Some of the articles recommended that further studies 'using qualitative methods would be able to investigate professionals' views of PAS and their perceived roles, including issues such as the degree and the wish for direct involvement'. ${ }^{37}$

\section{CONCLUSION}

This study has investigated the perspective of Australian pharmacists regarding their role in PAS and found the need for a sound ethical and legal framework to support PAS, as well as the need for training and budgetary considerations. These findings provide a platform to help shape future policies for the successful implementation of PAS in healthcare. Some of the findings have implications for clinical educators in developing new guidelines and policy-makers in formulating policies that detail the role of pharmacists in PAS. We recommend that these views and strategies be considered, and an inclusive approach be adopted for all stakeholders involved in this process.

Twitter Sami Isaac @sami_isaac_

Acknowledgements The researchers wish to thank the Australian pharmacists who participated in the study.

Contributors SI: writing, study design, data collection, data analysis (thematic coding), data interpretation, review and edits. AM: co-supervisor, data analysis, data interpretation, review and edits. BC: supervisor, data analysis, data interpretation, review and edits.

Funding SI is supported by a government-funded Research Training Program Stipend Scholarship during this study as part of his $\mathrm{PhD}$ candidature. The corresponding author had full access to all the data in the study and had final responsibility for the decision to submit for publication.

Competing interests None declared.

Patient consent for publication Not required.

Ethics approval This study was approved by the University of Sydney Human Research Ethics Committee (Ref No. 2017/624).

Provenance and peer review Not commissioned; externally peer reviewed.

Data availability statement All data relevant to the study are included in the article or uploaded as supplementary information.

Open access This is an open access article distributed in accordance with the Creative Commons Attribution Non Commercial (CC BY-NC 4.0) license, which permits others to distribute, remix, adapt, build upon this work non-commercially, and license their derivative works on different terms, provided the original work is properly cited, appropriate credit is given, any changes made indicated, and the use is non-commercial. See: http://creativecommons.org/licenses/by-nc/4.0/.

ORCID iDs

Sami Isaac http://orcid.org/0000-0002-9870-6129

Betty Chaar http://orcid.org/0000-0001-8757-9403

\section{REFERENCES}

1 Calabretta N. Consumer-driven, patient-centered health care in the age of electronic information. J Med Libr Assoc 2002;90:32-7.

2 Emanuel EJ, Onwuteaka-Philipsen BD, Urwin JW, et al. Attitudes and practices of euthanasia and physician-assisted suicide in the United States, Canada, and Europe. JAMA 2016;316:79-90.

3 Varadarajan R, Freeman RA, Parmar JR. Aid-in-Dying practice in Europe and the United States: legal and ethical perspectives for pharmacy. Research in Social and Administrative Pharmacy 2015;12:1-22.

4 Oxford University. Oxford English dictionary United Kingdom. Oxford University Press, 2017. https://en.oxforddictionaries.com/definition/ euthanasia

5 Deliens L, van der Wal G. The euthanasia law in Belgium and the Netherlands. The Lancet 2003;362:1239-40. 
6 Boudreau JD, Somerville MA. Euthanasia and assisted suicide: a physician's and ethicist's perspectives. Medicolegal and Bioethics 2014:4:1-12.

7 Happ MB, Capezuti E, Strumpf NE, et al. Advance care planning and end-of-life care for hospitalized nursing home residents. J Am Geriat Soc 2002;50:829-35.

8 Australian Human Rights Commission. Euthanasia, human rights and the law- issues paper Australia: Australian human rights Commission, 2016. Available: https://www.humanrights.gov.au/our-work/agediscrimination/publications/euthanasia-human-rights-and-law

9 Stevens CA, Hassan R, Death Mof. Management of death, dying and euthanasia: attitudes and practices of medical practitioners in South Australia. J Med Ethics 1994;20:41-6.

10 Baume P, O'Malley E. Euthanasia: attitudes and practices of medical practitioners. Med J Aust 1994:161:137-44.

11 Neil DA, Coady CAJ, Thompson J, et al. End-of-life decisions in medical practice: a survey of doctors in Victoria (Australia). $\mathrm{J} \mathrm{Med}$ Ethics 2007;33:721-5.

12 Beuthin R, Bruce A, Scaia M. Medical assistance in dying (MAID): Canadian nurses' experiences. Nurs Forum 2018;53:511-20.

13 Terkamo-Moisio A, Gastmans C, Ryynänen O-P, et al. Finnish nurses' attitudes towards their role in the euthanasia process. Nurs Ethics 2019;26:700-14.

14 Hanlon TRG, Weiss MC, Rees J. British community pharmacists views of physician-assisted suicide (PAS). J Med Ethics 2000;26:363-9.

15 Green J, Thorogood N. Qualitative methods for health research. Sage, 2013.

16 Glaser BG, Strauss AL. The discovery ofgrounded theory: Strategiesfor qualitative research. Chicago: Aldire, 1967.

17 Cica N. Euthanasia - the Australian Law in an International Context. In: Parliment of Australia. Australia: Parlimentary Library, 1996.

18 White BP, Willmott L, Ashby M. Palliative care, double effect and the law in Australia. Intern Med J 2011:41:485-92.

19 Australian Law Reform Commision (ALRC). Essentially Yours - The Protection of Human Genetic Information in Australia, Volume 1/2. Report 96 - 06. Ethical Considerations: Ethics in a regulatory framework Australia Southwood Press, 2003. Available: https://www. alrc.gov.au/publications/6-ethical-considerations/ethics-regulatoryframework\# ftn43

20 Isaac S, Chaar B, Savulescu J. Should pharmacists be allowed to Conscientiously object to medicines supply on the basis of their personal beliefs? Can J Hosp Pharm 2018;71.

21 Rutsohn P, Ibrahim N. An analysis of provider attitudes toward endof-life decision-making. Am J Hosp Palliat Care 2003;20:371-81.

22 Lee RY, Moles R, Chaar B. Mifepristone (RU486) in Australian pharmacies: the ethical and practical challenges. Contraception 2015;91:25-30.
23 Pharmaceutical Society of Australia. Code of ethics for pharmacists. care principle 2. Australia: Pharmaceutical Society of Australia, 2017.

24 Lawrence RE, Curlin FA. Autonomy, religion and clinical decisions: findings from a national physician survey. $J$ Med Ethics 2009;35:214-8.

25 Verweel L, Rosenberg-Yunger ZRS, Movahedi T, et al. Medical assistance in dying: examining Canadian pharmacy perspectives using a mixed-methods approach. Can Pharm J 2018;151:121-32.

26 Berard G, Walker J. Cpha surveys pharmacists on physician-assisted dying. Can Pharm J 2016;149:54-5.

27 Moreno J. Arguing euthanasia: the controversy over mercy killing, assisted suicide, and the" right to die": Simon and Schuster, 1995.

28 Oregon Department of Public Health. FAQs about the death with dignity act. Available: https://public.health.oregon.gov/ProviderPart nerResources/Evaluation-Research/DeathwithDignityAct/Pages/faqs. aspx

29 Meek C. Pharmacy involvement where assisted suicide and euthanasia are permitted. Pharm J 2006;277:614-5.

30 Termination of Life on Request and Assisted Suicide Act Netherlands, Article 1-24 2002.

31 Burki TK. Trends in euthanasia and assisted suicide. Lancet Oncol 2015;16:e433.

32 Emanuel EJ, Battin MP. What are the potential cost savings from legalizing physician-assisted suicide? N Engl J Med 1998;339:167-72.

33 International Anti-euthanasia Task force. brief to the Supreme Court: brief for amicus curiae.

34 Lau HS, Riezebos J, Abas V, et al. A nationwide study on the practice of euthanasia and physician-assisted suicide in community and hospital pharmacies in the Netherlands. Pharmacy World Science 2000;22:3-9.

35 Kirby T. Australian state of Victoria passes assisted dying bill. Lancet Respir Med 2018;6.

36 Bilsen J, Bauwens M, Bernheim J, et al. Physician-Assisted death: attitudes and practices of community pharmacists in East Flanders, Belgium. Palliat Med 2005;19:151-7.

37 Hackett EA, Francis S-A. "Death was a blessing" - should it ever be pharmaceutically hastened? British pharmacists' views. Pharm World Sci 2003;25:288-93.

38 Onwuteaka-Philipsen BD, Muller MT, van der Wal G. Euthanatics: implementation of a protocol to standardise euthanatics among pharmacists and GPs. Patient Educ Couns 1997;31:131-7.

39 Rupp MT, Isenhower HL. Pharmacists' attitudes toward physicianassisted suicide. Am J Hosp Pharm 1994:51:69-74.

40 Vivian J, Slaughter R, Calissi P. Michigan pharmacists' attitudes about medically assisted suicide. Journal of Michigan Pharmacy $1993 ; 31: 490-5$. 